Gastroenterologe $2010 \cdot 5: 403$

DOI 10.1007/s11377-010-0474-0

๑) Springer-Verlag 2010

R.M. Schmid

2. Medizinische Klinik, Klinikum rechts der Isar, Technische Universität München

\title{
Innovative Konzepte der Therapie gastrointestinaler Tumoren
}

fangreich; eine Altersbeschränkung wird nicht empfohlen.

- Beim Pankreaskarzinom sollten eine adjuvante Therapie nach R0-Resektion und eine additive Chemotherapie nach R1-Resektion erfolgen.

- Für die adjuvante Therapie des gastrointestinalen Stromatumors (GIST) ist der Tyrosinkinaseinhibitor Imatinib zugelassen.

Für die anderen Tumorentitäten des Gastrointestinaltrakts gibt es bisher keine schlüssige Datenlage. Ziel der adjuvanten Therapie ist die Reduktion des Risikos sowohl des Lokalrezidivs als auch der Fernmetastasierung.

Die Forschung der letzten Jahre hat unser Verständnis der molekularen Karzinogenese deutlich erweitert und Schaltermoleküle als Zielstrukturen für Therapieansätze identifiziert. Dies sind im Wesentlichen membranständige oder lösliche Kinasen, deren Aktivität entweder durch monoklonale Antikörper, wenn sie auf der Zelloberfläche lokalisiert sind, oder durch „small molecules“, die in die Tumorzellen eindringen, blockiert wird.

Trotz dieser vielversprechenden Entwicklungen ist die komplette Entfernung des Tumors (R0-Resektion) bei der lokalisierten Erkrankung die Therapie der Wahl. Sie kann aber nur bei einem Teil der Patienten durchgeführt werden.

- Für das Kolonkarzinom ist je nach postoperativem Tumorstadium und histopathologischem Befund eine adjuvante Therapie bei einer R0-Situation angezeigt. Die Datenlage ist um-

\section{( B Bei isolierten Lebermetastasen des kolorektalen Karzinoms sollte an einen kurativen Therapieansatz gedacht werden}

Das Lokalrezidiv stellt ein besonderes Problem des Rektumkarzinoms dar. Eine wesentliche Verbesserung wurde durch die präoperative Chemo- und Strahlentherapie erreicht. Für Magen- und Ösophaguskarzinome wird aufgrund der Datenlage eine perioperative Therapie favorisiert, da 50\% der Patienten trotz R0-Resektion innerhalb von 5 Jahren ein Lokalrezidiv erleiden oder Fernmetastasen entwickeln. Ziel ist die Reduktion der hohen Rezidivrate.

Obwohl im fortgeschrittenen Stadium eine systemische Ausbreitung vorliegt, kann beim kolorektalen Karzinom mit isolierten Lebermetastasen ein kurativer Therapieansatz erwogen werden. Hierbei muss ein multimodales Vorgehen erwogen werden. Ziel ist das Erreichen der Resektabilität bei initial grenzwertig resektablen isolierten Lebermetastasen. Lokalablative Verfahren kommen hier ebenso zum Einsatz.

Die Beiträge in dieser Ausgabe von Der Gastroenterologe geben einen sehr guten Überblick und zeigen moderne Konzepte der Therapie gastrointestinaler Tumoren auf. Die Datenlage ist für die verschiedenen Ansätze unterschiedlich dicht, daher ist die Behandlung von Patienten in Studien für die Weiterentwicklung sehr wichtig.

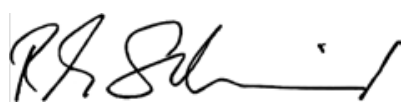

Roland M. Schmid

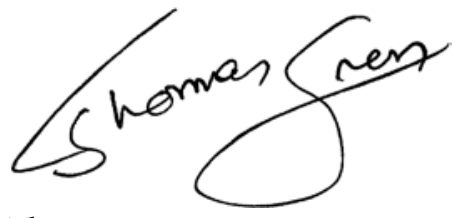

Thomas Gress

\section{Korrespondenzadresse \\ Prof. Dr. R.M. Schmid \\ 2. Medizinische Klinik, \\ Klinikum rechts der Isar, Technische \\ Universität München \\ Ismaninger Str. 22, \\ 81675 München \\ roland.schmid@lrz.tum.de}

